\title{
Estimating the Number of Errors In a System Using a Martingale Approach
}

\section{Paul Yip}

University of Hong Kong, Pokfulam

Key Words - Martingale difference, Removal experiment, Time-dependent failure intensity, Weight function, Zero-mean martingale

Summary \& Conclusions - A new, efficient procedure estimates the number of errors in a system. A known number of seeded errors are inserted into a system. The failure intensities of the seeded and real errors are allowed to be different and time dependent. When an error is detected during the test, it is removed from the system. The testing process is observed for a fixed amount of time $\tau$. Martingale theory is used to derive a class of estimators for the number of seeded errors in a continuous time setting. Some of the estimators and their associated standard deviations have explicit expressions. An optimal estimator among the class of estimators is obtained. A simulation study assesses the performance of the proposed estimators.

\section{INTRODUCTION}

Mills [15] proposed a capture-recapture sampling method which allows estimation of the number of errors in a system by randomly inserting a known number of errors and then testing the system for both inserted \& indigenous errors. Duran \& Wiorkowski [5], and Yip \& Fong [21] derived maximum likelihood estimates of the indigenous errors and showed methods to obtain $s$-confidence limits. They assumed that the seeded errors behave as if randomly selected from the distribution of possible real errors in the system. The error seeding method was also discussed in $[2,16]$.

Martingale theory is used here for an alternative estimation procedure which allows different failure intensities for the seeded \& real errors. In order to avoid the identifiability problem, a known constant proportionality is assumed between the seeded \& real errors. This problem can be related to a removal experiment for a closed population of a certain type of animal in wildlife studies. Several authors have addressed this problem $[4,6,9-11,13,14]$. Martingale theory is applied in reliability studies in $[12,19]$.

Martingale theory is used to derive a class of estimators for the population size of the real errors. Some of the estimators and their associated standard deviations have explicit expressions. An optimal estimator among the class estimators for the number of real errors is obtained.

Acronyms \& Abbreviations ${ }^{I}$

RMSE (square) root of mean square deviation

\author{
StdDev standard deviation \\ ZMM zero-mean martingale.
}

Notation

$\mathcal{F}_{t} \quad$ history of the process during $[0, t]$

$\nu \quad$ number of real errors in the system (parameter of interest)

$D \quad$ number of seeded errors (known)

$\lambda_{t}, \beta_{t}$ failure intensity for [real, seeded] errors

$U_{u-}, M_{u-}$ number of [real, seeded] errors detected/removed in $[0, u)$

$U_{t}, M_{t}$ number of [real, seeded] errors detected/removed in $[0, t]$

$\mathcal{U}_{t}, \mathfrak{N}_{t}$ zero-mean martingales

$R_{u} \quad$ see (3)

$\theta \quad \lambda_{t} / \beta_{t}$, a known constant

$\operatorname{Av}_{1}(\hat{\nu}), \mathrm{SD}_{1}(\hat{\nu})$ [average, StdDev] of the 500 simulated values $\stackrel{d}{=} \quad$ distributed as.

Other, standard notation is given in "Information for Readers \& Authors" at the rear of each issue.

\section{Assumptions}

1. A known number of errors, $D$, is seeded in the system at the beginning of the experiment.

2. $\lambda_{t} \& \beta_{t}$ may be time dependent.

3. $\theta$ is a known constant; it need not be 1 .

4. The same failure intensity is applied to each type of error in the system.

5a. Errors are removed (without introducing new errors or affecting old errors) immediately after detection.

5b. Errors are detected/removed one at a time. (This allows a continuous time formulation.)

6. $U_{u-}, M_{u-}$ are measurable w.r.t. $\mathcal{F}_{u-}$.

\section{CONTINUOUS-TIME MARTINGALE}

The methods here are based on results for continuous martingales and follow from the work of [1]. The appendix informally explains martingales. For this paper, ZMM is a stochastic process $\left\{\mathfrak{T}_{t} ; t \geq 0\right\}$ such that:

$\mathrm{E}\left\{\mathfrak{N}_{0}\right\}=0$,

$\mathrm{E}\left\{\left|\mathfrak{M}_{t}\right|\right\}<\infty$, for all $t \geq 0$,

\footnotetext{
${ }^{I}$ The singular \& plural of an acronym or abbreviation are always spelled the same.
} 
$\mathrm{E}\left\{\mathfrak{N}_{t+h} \mid \mathfrak{F}_{t}\right\}=\mathfrak{N}_{t}$, for all $t \geq 0, h>0$

We can construct $\mathrm{ZMM}$ with respect to the counting processes $U_{t} \& M_{t}$. The following 2 statements are true:

$d U_{t} \mid \mathcal{F}_{t-} \stackrel{d}{=} \operatorname{binm}\left(\lambda_{t} \cdot d t, \nu-U_{t-}\right)$

$d M_{t} \mid F_{t-} \stackrel{d}{=} \operatorname{binm}\left(\beta_{t} \cdot d t, D-M_{t-}\right)$.

By the Doob-Meyer decomposition, (1) \& (2) are ZMM with respect to the increasing family of $\sigma$-fields or filtration $\left\{\mathfrak{F}_{t}, t\right.$ $\geq 0\}$

$u_{t}=U_{t}-\int_{0}^{t} \lambda_{u} \cdot\left(\nu-U_{u-}\right) d u$,

$\mathfrak{N}_{t}=M_{t}-\int_{0}^{t} \beta_{u} \cdot\left(D-M_{u-}\right) d u$

In the presence of $\lambda_{t}$, identifiability problems occur when we want to estimate $\nu$ by (1) only. Obviously, the information of $\nu \& \lambda_{t}$ in (1) is confounded. The extra effort by inserting $D$ errors into the system in the beginning of the testing provides an extra equation to estimate $\nu$. However an identifiability problem can still occur with an extra unknown parameter $\beta$; thus assumption 3 is needed. Sacks \& Chiang [18] and Wolter [21] made a similar assumption of constant proportionality between two intensities in a competing risk model and a capturerecapture experiment respectively.

We now use the ZMM of (1) \& (2) to get an estimating equation which can generate a class of estimators for the population size of real errors, $\nu$. Define a martingale-difference:

$d \mathbb{R}_{u}=\left(D-M_{u-}\right) \cdot d U_{u}-\theta \cdot\left(\nu-U_{u-}\right) \cdot d M_{u}$

Then $\mathrm{E}\left\{d R_{u} \mid \mathscr{F}_{u-}\right\}=0$.

\section{Notation}

$W_{u-} \quad$ a measurable function w.r.t. $\mathcal{F}_{u-}$

$\mathbb{R}_{i}^{*} \quad \int_{0}^{t} W_{u-} \cdot\left(D-M_{u-}\right) d U_{u}-\theta \cdot \int_{0}^{t} W_{u-} \cdot\left(\nu-U_{u-}\right) d M_{u}$.

$\mathfrak{R}^{*}=\left\{\mathfrak{R}_{t}^{*}, t \geq 0\right\}$, is a $\mathrm{ZMM}$.

Equate (3b) to zero and evaluate it at time $\tau$; then a class of estimators for $\nu$ is obtained:

$$
\begin{aligned}
\hat{\nu}_{w} & =\left[\int_{0}^{\tau} W_{u-} \cdot\left(D-M_{u-}\right) d U_{u}+\theta \cdot \int_{0}^{\tau} W_{u-} \cdot U_{u-} d M_{u}\right] \\
& /\left[\theta \cdot \int_{0}^{\tau} W_{u-} d M_{u}\right],
\end{aligned}
$$

which depends on the choice of $W_{u-}$. The conditional variance of $R_{\tau}$ is:

$$
\begin{aligned}
& \operatorname{Var}\left\{\mathbb{R}_{\tau}^{*}\right\}=\mathrm{E}\left\{\int_{0}^{\tau} W_{u-}^{2} \cdot\left(D-M_{u-}\right)^{2} d U_{u}+\theta^{2} \cdot \int_{0}^{\tau} W_{u-}^{2}\right. \\
& \left.\cdot\left(\nu-U_{u-}\right)^{2} d M_{u}\right\} .
\end{aligned}
$$

The terms for variance follow from a standard result, eg, [3]; the covariance is zero by virtue of the orthogonality result of martingales since $d M_{u} \& d U_{u}$ cannot jump simultaneously. Use a result from [17]:

$\mathbb{R}_{t}^{*} / \sqrt{\mathbb{R}_{t}^{*}} \rightarrow N(0,1)$ as $\nu, D \rightarrow \infty$.

$$
\begin{gathered}
\operatorname{StdDev}\left\{\hat{\nu}_{w}\right\}=\left[\int_{0}^{\tau} W_{u-}^{2} \cdot\left(D-M_{u-}\right)^{2} d U_{u}+\theta^{2}\right. \\
\left.\cdot \int_{0}^{\tau} W_{u-}^{2} \cdot\left(\hat{\nu}_{w}-U_{u^{-}}\right)^{2} d M_{u}\right]^{1 / 2} /\left[\theta \cdot \int_{0}^{\tau} W_{u-} d M_{u}\right] .
\end{gathered}
$$

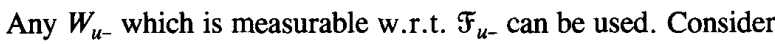
some choices of $W_{u-}$ which gives explicit expression for $\nu$.

Choice 1: $\mathrm{W}_{\mathrm{u}-}=1$

$$
\begin{aligned}
& \hat{\nu}_{1}=\left[\int_{0}^{\tau}\left(D-M_{u-}\right) d U_{u}+\theta \cdot \int_{0}^{\tau} U_{u-} d M_{u}\right] /\left[\theta \cdot M_{\tau}\right], \\
& \operatorname{StdDev}\left\{\hat{\nu}_{1}\right\}=\left[\int_{0}^{\tau}\left(D-M_{u-}\right)^{2} d U_{u}+\theta^{2}\right. \\
& \left.\quad \cdot \int_{0}^{\tau}\left(\nu_{1}-U_{u-}\right)^{2} d M_{u}\right]^{1 / 2} /\left[\theta \cdot M_{\tau}\right] .
\end{aligned}
$$

Choice 2: Quasi-Score $W_{u-}$

An optimal estimating function within such a class of martingale estimating functions in (3) is discussed in $[7,8]$. The best choice, quasi-score, is:

$W_{u-}=\left(d R_{u}\right) /\left(d\langle R\rangle_{u}\right)$

$$
\begin{aligned}
& d \mathbb{R}_{u}=\mathrm{E}\left\{d \mathbb{R}_{u} / d \nu \mid F_{u-}\right\}=\mathrm{E}\left\{\theta \cdot d M_{u} \mid F_{u-}\right\} \\
&= \theta \cdot\left(D-M_{u-}\right) \cdot \beta_{u} \cdot d u \\
& d\left\langle(\mathcal{R}\rangle_{u}=\mathrm{E}\left\{\left(d \mathbb{R}_{u}\right)^{2} \mid F_{u-}\right\}\right. \\
&=\mathrm{E}\left\{\left(D-M_{u^{-}}\right)^{2} \cdot d U_{u}+\theta^{2} \cdot\left(\nu-U_{u-}\right)^{2} \cdot d M_{u} \mid F_{u-}\right\} \\
&=\left(D-M_{u-}\right)^{2} \cdot\left(\nu-U_{u-}\right) \cdot \lambda_{u} d u+\theta^{2} \cdot\left(\nu-U_{u-}\right)^{2} \\
& \quad \cdot\left(D-M_{u-}\right) \cdot \beta_{u} \cdot d u .
\end{aligned}
$$

Hence the optimal weight corresponding to (3) is:

$W_{u_{-}}^{*}=\left[\left(\nu-U_{u_{-}}\right) \cdot\left[\left(D-M_{u_{-}}\right)+\left(\nu-U_{u_{-}}\right) \cdot \theta\right]\right]^{-1}$. 
Accordingly, the optimal estimating equation (gives the tightest $s$-confidence limits for $\hat{\nu}$ ) is, using (9a):

$\mathbb{R}_{t}^{*}=\int_{0}^{t} W_{u-}^{*} \cdot\left(D-M_{u-}\right) d U_{u}-\theta \cdot \int_{0}^{t} W_{u-}^{*} \cdot\left(\nu-U_{u-}\right) d M_{u}$

$\hat{\nu}^{*}$ is the solution of (9b). An explicit expression is not available; an iterative procedure is required. From (5),

$$
\begin{gathered}
\operatorname{StdDev}\{\hat{\nu}\}=\left[\int_{0}^{t}\left(D-M_{u_{-}}\right)^{2} / \psi_{u-}^{2} d U_{u}+\theta^{2}\right. \\
\left.\cdot \int_{0}^{t}\left(\hat{\nu}-U_{u_{-}}\right)^{2} / \psi_{u-}^{2} d M_{u}\right] /\left[\theta \cdot \int_{0}^{t} d M_{u} / \psi_{u^{-}}\right] \\
\psi_{u_{-}} \equiv\left(\hat{\nu}^{*}-U_{u_{-}}\right) \cdot\left[\left(D-M_{u_{-}}\right)+\left(\hat{\nu}^{*}-U_{u_{-}}\right) \cdot \theta\right]
\end{gathered}
$$

\section{SIMULATION}

A Monte Carlo simulation was performed to evaluate the performance of $\hat{\nu}_{1}$ from (7) and $\hat{\nu}^{*}$ from (9b). Various values of $\theta$ have been used. An arbitrary stopping time could be used for $\tau$. Here we assume that the stopping time is determined by the removed proportion of the seeded errors. We investigate the effects of $\theta$, the stopping time, and the proportion of seeded errors placed in the system, on the performance of the estimators.

The simulation results are in two tables. Table 1 lists all the sets of parameter values in the simulation study. Table 2 lists the results of the 7 trials in table 1 . There were 500 repetitions for each trial.

\section{Notation}

$\operatorname{Av}_{2}\left(\operatorname{SD}_{1}(\hat{\nu})\right)$ average $\operatorname{StdDev}\{\hat{\nu}\}$ of the 500 simulated trials $\operatorname{RMSE}\{\hat{\nu}\} \quad\left[\operatorname{Bias}(\hat{\nu})^{2}+\operatorname{SD}_{1}(\hat{\nu})^{2}\right]^{1 / 2}$

$\operatorname{Bias}\{\hat{\nu}\} \hat{\nu}-\nu$

Coverage proportion of the estimates between the $95 \%$ $s$-confidence limits

$P \quad$ proportion of seeded errors removed: the stopping criterion.

The statistics computed were:

$\operatorname{Av}_{1}(\hat{\nu}), \mathrm{SD}_{1}(\hat{v}), \mathrm{Av}_{2}\left(\mathrm{SD}_{1}(\hat{\nu})\right), \mathrm{RMSE}$, Coverage.

- Trials 1 - 3 examine the role of $P$ and the performance of the estimators when $\nu, D, \theta$ are kept the same. The estimators should perform better when $P$ is large since more information has been collected.

- Trials 2, 4, 5 examine the effect of $\theta$ when $\nu, D, P$ are kept the same. When $\theta>1$, the performance of the estimators should improve since more real errors are removed.
- Trials 4, 6, 7 examine the effect of $\nu$ on the performance when $D, P$ are kept the same.

TABLE 1

Description of the Trials

\begin{tabular}{llllll}
\hline Trial & $\nu$ & $D$ & $D / \nu$ & $\theta$ & $P$ \\
\hline 1 & 400 & 50 & 0.125 & 0.5 & 0.3 \\
2 & 400 & 50 & 0.125 & 0.5 & 0.5 \\
3 & 400 & 50 & 0.125 & 0.5 & 0.9 \\
4 & 400 & 50 & 0.125 & 1.0 & 0.5 \\
5 & 400 & 50 & 0.125 & 2.0 & 0.5 \\
6 & 100 & 50 & 0.5 & 1.0 & 0.5 \\
7 & 1000 & 50 & 0.05 & 1.0 & 0.5 \\
\hline
\end{tabular}

Table 2. Simulation Results ${ }^{\dagger}$ for the Trials in Table 1 [The top row is for $\hat{\nu}_{1}$; the bottom row is for $\hat{\nu}^{*}$ ]

\begin{tabular}{lcrrrrrr}
\hline Trial & \multicolumn{1}{c}{$\nu$} & \multicolumn{1}{c}{$D$} & $\mathrm{Av}_{1}(\hat{\nu})$ & $\mathrm{SD}_{1}(\hat{\nu})$ & $\mathrm{Av}_{2}\left(\mathrm{SD}_{1}(\hat{\nu})\right)$ & $\mathrm{RMSE}$ & Coverage \\
\hline 1 & 400 & 50 & 402.30 & 104.05 & 192.10 & 216.94 & 1.00 \\
& & & 397.69 & 102.08 & 103.87 & 139.78 & 0.95 \\
2 & 400 & 50 & 403.52 & 72.94 & 140.26 & 156.85 & 1.00 \\
& & & 399.10 & 70.86 & 74.18 & 98.90 & 0.99 \\
3 & \multirow{2}{*}{400} & 50 & 402.38 & 50.14 & 87.63 & 99.98 & 1.00 \\
& & & 396.10 & 39.85 & 36.91 & 52.07 & 0.92 \\
4 & \multirow{2}{*}{400} & 50 & 401.23 & 66.46 & 64.66 & 88.80 & 0.95 \\
& & & 393.27 & 61.51 & 58.04 & 81.60 & 0.93 \\
5 & \multirow{2}{*}{400} & 50 & 400.29 & 53.38 & 36.88 & 60.27 & 0.80 \\
& & & 388.54 & 40.54 & 35.69 & 52.04 & 0.91 \\
6 & \multirow{2}{*}{100} & 50 & 99.32 & 18.59 & 18.52 & 25.19 & 0.94 \\
& & & 96.82 & 16.21 & 16.47 & 22.56 & 0.96 \\
7 & 1000 & 50 & 999.31 & 153.94 & 154.28 & 209.65 & 0.95 \\
& & & 928.02 & 88.66 & 129.19 & 165.83 & 0.95 \\
\hline
\end{tabular}

${ }^{\dagger}$ The number of significant figures is not intended to imply any accuracy in the estimates, but to illustrate the arithmetic.

- Trials 1 - 3 confirmed that when $P$ is large: the performance of $\hat{\nu}_{1} \& \hat{\nu}^{*}$ improve, and the RMSE \& $\operatorname{SD}(\hat{\nu})$ decrease appreciably.

- Trials $2,4,5$ showed that the performance of $\hat{\nu}_{1} \& \hat{\nu}^{*} \mathrm{im}$ prove when $\theta>1$.

- Trials 4, 6, 7 showed that: $\hat{\nu}^{*}$ underestimates $\nu$ when $\nu$ is large.

For all trials,

- $\hat{\nu}_{1}$ is satisfactory though $\operatorname{RMSE}\left(\hat{\nu}_{1}\right)>\operatorname{RMSE}\left(\hat{\nu}^{*}\right)$,

- Coverage $\left(\hat{\nu}_{1}\right)$ is approx $80 \%-100 \%$, Coverage $\left(\hat{\nu}^{*}\right)$ is approx $91 \%-99 \%$,

- performance of $\hat{\nu}^{*}$ is uniformly better than $\hat{\nu}_{1}$.

- $\hat{\nu}_{1}$ is easier to compute and can be used as an initial value to search for $\hat{\nu}^{*}$. 


\section{ACKNOWLEDGMENT}

I am pleased to thank the Associate Editor and referees for their comments which improved the presentation of the paper. The work was supported by the Hong Kong Research Grant Council.

\section{APPENDIX}

Counting Process, Martingales, and Stochastic Integrals

$N=\left\{N_{t} ; t \in J\right\}$ is a counting process if it begins at 0 and increases only by integer-valued jumps, where $\mathfrak{I}=[0, \infty)$. The observed process can include one or more counting processes, such as the process counting the number that have failed and the process counting the number that have been censored (lost to follow-up). The increasing family of histories $\mathfrak{F}=\left\{\mathscr{F}_{t}\right.$; $t \in J\}$ is a filtration.

A process $\mathfrak{N}=\left\{\mathscr{N}_{t} ; t \in \mathfrak{I}\right\}$ is a martingale (with respect to $\mathcal{F}$ ) if, for all $t \in \mathfrak{J}$ :

$\mathrm{E}\left\{\left|\mathfrak{T}_{t}\right|\right\}<\infty$

$\mathrm{E}\left\{\mathfrak{M}_{t+s} \mid \mathfrak{F}_{t}\right\}=\mathfrak{M}_{t}$ for all $s \in \mathfrak{J}$.

A consequence of the martingale property is that $\mathrm{E}\left\{\mathfrak{T}_{t}\right\}=$ $\mathrm{E}\left\{\mathfrak{T}_{0}\right\}$ for all $t \in \mathcal{J}$. For a ZMM, $\mathrm{E}\left\{\mathfrak{T}_{0}\right\}=0$.

This appendix introduce some properties of martingales which are useful to reliability analysts and gives results for continuous-time martingales in the context of independent continuous failures times. These results are most relevant to applications in this paper. These are not always the most general results. A more rigorous approach is in [3].

\section{Notation}

$N_{t} \quad$ number of the $n$ failure times falling in $(0, t]$ $Y_{s} n-N_{s-}:$ number still at risk just prior to time $s$.

Let $N_{0}=0$. Partition the interval $(0, t]$ into many very small increments. Then write $N_{t}$ as $\int_{0}^{t} d N_{s}$. We are concerned with the conditional distribution of $d N_{s}$, given $\mathfrak{F}_{s-}$. This conditional distribution is binomial:

$d N_{s} \mid \mathcal{F}_{s-} \stackrel{d}{=} \operatorname{binm}\left(\alpha_{s} \cdot d s, Y_{s}\right)$

Most time increments contain 0 failures and a small fraction of the increments contain 1 failure; the probability of an increment containing more than 1 failure is negligible. As,

$\mathrm{E}\left\{d N_{s} \mid \mathcal{F}_{s-}\right\}=Y_{s} \cdot \alpha_{s} \cdot d s$, then,

$d \mathscr{T}_{s}=d N_{s}-Y_{s} \cdot \alpha_{s} \cdot d s$

can be treated as a martingale-difference. It follows that the process,
$\mathfrak{M}=\left\{\mathfrak{T}_{t} ; t \in \mathfrak{I}\right\}$, specified by

$\mathfrak{N}_{t}=\int_{0}^{t} d \mathscr{N}_{s}=N_{t}-\int_{0}^{t} Y_{s} \cdot \alpha_{s} d s$,

is a $\mathrm{ZMM}$.

Let $W=\left\{W_{t} ; t \in J\right\}$ be any process such that $W_{s-}$ is determined by $\mathcal{F}_{s-}$ for each $s \geq 0$, then each,

$W_{s-} \cdot d \mathfrak{N}_{s}$

is also a martingale-difference. It follows that $\mathfrak{T}^{*}$ specified by,

$\mathfrak{M}_{t}^{*}=\int_{0}^{t} W_{s-} d \mathbb{M}_{s}=\int_{0}^{t} W_{s-} d N_{s}-\int_{0}^{t} W_{s-} \cdot Y_{s} \cdot \alpha_{s} d s$,

is a ZMM. This property is very useful for generating estimating equations. In a similar manner, using various choices of $W$, one obtains estimating equations for other quantities.

The next step is to associate a standard deviation with such an estimate.

$$
\begin{aligned}
\operatorname{Var} & \left\{W_{s-} \cdot d \mathfrak{M}_{s}\right\}=\mathrm{E}\left\{\operatorname{Var}\left\{W_{s-} \cdot d \mathfrak{N}_{s} \mid \mathscr{F}_{s-}\right\}\right\}+\operatorname{Var}\left\{\mathrm { E } \left\{W_{s-}\right.\right. \\
& \left.\left.\cdot d \mathfrak{N}_{s} \mid \mathfrak{F}_{s-}\right\}\right\} \\
& =\mathrm{E}\left\{W_{s-}^{2} \cdot \operatorname{Var}\left\{d \mathfrak{N}_{s} \mid \mathfrak{F}_{s-}\right\}\right\}=\mathrm{E}\left\{W_{s-}^{2} \cdot \operatorname{Var}\left\{d N_{s} \mid F_{s-}\right\}\right\} \\
& =\mathrm{E}\left\{W_{s-}^{2} \cdot Y_{s} \cdot \alpha_{s} \cdot d s \cdot\left(1-\alpha_{s} \cdot d s\right)\right\} \approx \mathrm{E}\left\{W_{s-}^{2} \cdot Y_{s} \cdot \alpha_{s} \cdot d s\right\}
\end{aligned}
$$

Hence,

$\operatorname{Var}\left\{\Re_{t}^{*}\right\}=\mathrm{E}\left\{\int_{0}^{\mathrm{t}} \mathrm{W}_{\mathrm{s}-}^{2} \cdot Y_{s} \cdot \alpha_{s} d s\right\}=\mathrm{E}\left\{\int_{0}^{t} W_{s-}^{2} d N_{s}\right\}$.

A central limit theorem also applies, indicating that inference can be made by using the $s$-normal distribution when the amount of data is sufficiently large [3].

\section{REFERENCES}

[1] O.O. Aalen, "Weak convergence of stochastic integral related to counting processes", Z. Wahrverw. Geb., vol 38, 1977, pp 261-277.

[2] J.C. Ammann, P.E. Ammann, "An experimental evaluation of simple methods for seeding program errors', Proc. $7^{\text {th }}$ Int'l Conf. Software Eng'g, 1985, pp 337-342.

[3] P.K. Andersen, O. Borgan, "Counting process models for life history data", Scand. J. Statistics, vol 12, 1985, pp 97-158.

[4] S. Blumenthal, R. Marcus, "Estimating population size with exponential failure”, J. Amer. Statistical Assoc, vol 70, 1975, pp 913-922.

[5] J.W. Duran, J.J. Wiorkowski, "Capture-recapture sampling for estimating software content', IEEE Trans. Software Eng'g, vol SE-7, 1981, pp 147-148.

[6] E.H. Forman, N.D. Singpurwalla, "An empirical stopping rule for debugging and testing computer software", J. Amer. Statistical Assoc, vol 72, 1977, pp 750-757.

[7] V.P. Godambe, "The foundations of finite sample estimation in stochastic processes", Biometrika, vol 72, 1985, pp 419-428. 
[8] V.P. Godambe, C.C. Heyde, "Quasi-likelihood and optimal estimation", Int'l Statistics Rev, vol 55, 1987, pp 231-244.

[9] I.B.J. Goudie, C.M. Goldie, "Initial size estimation for the linear pure death process", Biometrika, vol 68, 1981, pp 543-550.

[10] H. Joe, N. Reid, "Estimating the number of faults in a system", J. Amer. Statistical Assoc, vol 80, 1985, pp 222-226.

[11] H. Joe, N. Reid, "On the software reliability models of Jelinski-Moranda and Littlewood"', IEEE Trans. Reliability, vol R-34, 1985, pp 216-218.

[12] G. Koch, P.J.C. Spreij, " Software reliability as an application of martingale \& filtering theory", IEEE Trans. Reliability, vol R-32, 1983, pp 342-345.

[13] B. Littlewood, J.L. Verrall, "Likelihood function of a debugging model for computer software reliability', IEEE Trans. Reliability, vol R-30, 1981, pp 145-148.

[14] R.J. Meinhold, N.D. Singpurwalla, "Bayesian analysis of a commonly used model for describing software failures", Statistician, vol 32, 1983, pp 168-73.

[15] H.D. Mills, "On the statistical validation of computer programs", IBM FSD Rep, 1970 Jul.

[16] M. Ohba, "SPWL: Improvement of error seeding method", Reliability Theory and Applications (S. Osaki, J. Cai, Eds), 1987, pp 294-303; World Scientific, Singapore

[17] R. Rebolledo, "Central limit theorem for local martingales", Z. Wahr. verw. Geb., vol 51, 1980, pp 269-286.

[18] S.T. Sacks, C.L. Chiang, "A transition probability model for the study of chronic disease", Math. Bioscience, vol 34, 1977, pp 325-346.
[19] M.C. van Pul, "Asymptotic properties of a class of statistical models in software reliability", Scand. J. Statistics, vol 19, 1992, pp 235-253.

[20] K.M. Wolter, "Capture-recapture estimation in the presence of a known sex ratio", Biometrics, vol 46, 1990, pp 157-162.

[21] P. Yip, D.Y.T. Fong, "Estimating population size from a removal experiment in discrete time", Statist. and Prob. Letters, vol 16, 1993, pp 129-135.

\section{AUTHOR}

Dr. Paul Yip; Dept. of Statistics; Univ. of Hong Kong; Pokfulam Road; HONG KONG.

e-mail: sfpyip@hkucc.hku.hk

Dr. Yip graduated from the University of Melbourne; he received a $\mathrm{PhD}$ (1988) in Statistics from La Trobe University, Australia. He is a senior lecturer in the Department of Statistics at the University of Hong Kong. His research interests are reliability studies and mathematical modeling of biological \& ecological systems. He is a member of the International Group of Application of Capture-Recapture Techniques in Epidemiology.

Manuscript received 1994 February 2.

IEEE Log Number 94-11254
OPINION ITEMS OPINION ITEMS OPINION ITEMS

Do you want to sound off on a reliability topic? Then DO IT! Write a "Letter to the Editor".

The Editorial Staff welcomes your comments on material published in this Transactions (eg, editorials and technical papers) as well as on other topics of interest to Transactions readers. If you comment on the work of someone else, that person will be invited to present a
OPINION ITEMS OPINION ITEMS OPINION ITEMS

reply or clarification. We will publish controversy, but we don't want to publish misunderstandings.

We encourage interaction between you and the original author to minimize misunderstanding and clarify the areas of disagreement.

Material judged unsuitable as a refereed paper can sometimes be abbreviated and published as opinion. Opinions will be edited as to length and propriety - at the sole discretion of the Editorial staff. 